\title{
Multisensory Interaction in Saccadic Reaction Time: A Time-Window-of-Integration Model
}

\author{
Hans Colonius ${ }^{1}$ and Adele Diederich ${ }^{2}$
}

\begin{abstract}
- Saccadic reaction time to visual targets tends to be faster when stimuli from another modality (in particular, audition and touch) are presented in close temporal or spatial proximity even when subjects are instructed to ignore the accessory input (focused attention task). Multisensory interaction effects measured in neural structures involved in saccade generation (in particular, the superior colliculus) have demonstrated a similar spatio-temporal dependence. Neural network models of multisensory spatial integration have been shown to generate convergence of the visual, auditory, and tactile reference frames and the sensorimotor coordinate
\end{abstract}

\section{INTRODUCTION}

Saccades are fast, voluntary movements of the eyes to align the high-resolution fovea with objects and events of interest. In a natural environment, saccades are part of a rapid goal-directed orienting response, often including head movements, to stimuli occurring in the periphery. These stimuli are usually crossmodal (e.g., a rapidly approaching subway train). In addition to visual and auditory inputs, vestibular and somatosensory afferents have access to the saccade-generating mechanism. Thus, it is no surprise that the oculomotor system has become a prominent arena for the analysis of multisensory integration. For example, it has been found that saccadic reaction time to visual targets (the time between the onset of the visual stimulus and the onset of the saccadic eye movement) tends to be faster when auditory stimuli are presented in close temporal or spatial proximity (Colonius \& Arndt, 2001; Harrington \& Peck 1998; Hughes, Nelson, \& Aronchick, 1998; Frens, Van Opstal, \& Van der Willigen, 1995). Similar response enhancement effects for saccades have been observed for combining visual and somatosensory stimuli (cf. Groh \& Sparks, 1996a, for monkeys; Amlôt, Walker, Driver, \& Spence, 2003; Diederich, Colonius, Bockhorst, \& Tabeling, 2003, for humans).

These psychophysical observations are in line with neurophysiological evidence for multisensory integration in the deep layers of the superior colliculus (SC),

${ }^{1}$ Universitaet Oldenburg, ${ }^{2}$ International University Bremen transformations necessary for coordinated head and eye movements. However, because these models do not capture the temporal coincidences critical for multisensory integration to occur, they cannot easily predict multisensory effects observed in behavioral data such as saccadic reaction times. This article proposes a quantitative stochastic framework, the time-window-of-integration model, to account for the temporal rules of multisensory integration. Saccadic responses collected from a visual-tactile focused attention task are shown to be consistent with the time-window-of-integration model predictions.

an area clearly involved in saccade generation (Robinson, 1972). Multisensory neurons in SC of anesthetized cats (Meredith \& Stein, 1986a, 1986b; Stein, MagalhaesCastro, \& Kruger, 1976) and monkeys (Wallace, Wilkinson, \& Stein, 1996) show an enhanced response to particular combinations of visual, auditory, and tactile stimuli paralleling the spatial-temporal rules observed in behavioral studies. Similar results have recently been obtained for recordings from unanesthetized cats by Wallace, Meredith, and Stein (1998) and from the awake behaving monkey by Bell, Corneil, Meredith, and Munoz (2001) and by Frens and Van Opstal (1998). Information about stimulus location is represented topographically within the structure by an orderly arrangement of neurons according to the location of their respective receptive fields (RFs). The spatial register among the different sensory maps is formed by multisensory neurons whose different RFs are in register with one another (Meredith \& Stein, 1986a, 1986b; for a review, see Stein \& Meredith, 1993). In addition, the SC contains a motor map composed of output neurons coding appropriate eye movements (Sparks, 1986), that is, the locus of activity in the motor map encodes a movement command that reorients the eyes (and the head) a given distance in a particular direction.

There are a number of explicit neural network models of multisensory spatial integration mechanism describing the integration of visual, auditory, and tactile reference frames and the sensorimotor coordinate transformations necessary for directed head and eye movements (cf. Deneve, Latham, \& Pouget, 2001; Xing \& 
Andersen, 2000). Although these models capture many aspects of spatial rules of multisensory integration (for a recent critical review, see Pouget, Deneve, \& Duhamel, 2002), they do not address the effects of temporal coincidences of crossmodal stimulus sets. In this article, we propose a time-window-of-integration (TWIN) model to serve as a framework incorporating the temporal rules of multisensory integration in saccades. A parametric version of the model is fit to a recent set of data on visual-tactile interaction in saccades.

\section{TWIN Model}

The classic explanation for a speed-up of responses to multisensory stimuli is that subjects are merely responding to the first stimulus detected. Formally, observed reaction time (RT) would then be the minimum of the response time to the visual, auditory, or tactile signal causing a statistical facilitation effect (Raab, 1962). However, several studies have clearly shown that statistical facilitation is not sufficient to explain the speedup in saccadic reaction time (Harrington \& Peck, 1998; Hughes, Reuter-Lorenz, Nozawa, \& Fendrich, 1994; Hughes et al., 1998; Corneil \& Munoz, 1996). Using Miller's inequality as a benchmark test (see Diederich, 1995; Colonius, 1990; Miller, 1982), responses to bimodal stimuli have been found to be faster than predicted by statistical facilitation, in particular, when the stimuli were spatially aligned. Moreover, the race model has no simple explanation for the decrease in facilitation observed with increasing spatial disparity between the stimuli.

Although there is increasing evidence for a complex network of largely parallel neural subprocesses underlying performance even in simple crossmodal tasks (Driver \& Spence, 2000), the initial separation of the afferent pathways for the different sensory modalities suggests that one can distinguish at least two serial stages of saccadic reaction time: an early, afferent stage of peripheral processing (first stage) followed by a compound stage of converging subprocesses (second stage). As shown below, in conjunction with a number of weak additional assumptions, some interesting and empirically testable predictions can be derived from this simple setup.

Even under invariant experimental conditions, saccadic responses typically vary from one trial to the next due to an inherent variability of the underlying neural processes in both ascending and descending pathways. This is taken into account in the TWIN model by assuming the duration of each of the stages to be a random variable (Figure 1).

\section{First Stage Assumption}

In the first stage there is a race among the peripheral neural excitations in the visual, auditory, and/or somatosensory pathways triggered by a crossmodal stimulus complex.
Because the first stage refers to very early sensory processing, random processing times for visual, auditory, and somatosensory stimuli are assumed to be statistically independent.

\section{Second Stage Assumption}

The second stage comprises neural integration of the input and preparation of an ocular motor response. Multisensory integration manifests itself in an increase or decrease of second stage processing time.

Distinguishing between two stages only is clearly an oversimplification. But note that the second stage is defined by default: It includes all subsequent, possibly overlapping, processes that are not part of the peripheral processes in the first stage (for a formal description of the model, see Methods section).

\section{TWIN Assumption}

Multisensory integration occurs only if the peripheral processes of the first stage all terminate within a given time interval, the "window of integration".

The window of integration acts like a filter determining whether afferent information delivered from different sensory organs is registered close enough in time to allow for multisensory integration. Passing the filter is a necessary, but not a sufficient, condition for multisensory integration to occur. The reason is that multisensory integration also depends on the spatial configuration of the stimuli. However, rather than assuming the existence of a joint spatial-temporal window of integration permitting interaction to occur only for both spatially and temporally neighbored stimuli, the TWIN model allows for multisensory integration to occur even for rather distant stimuli (of different modalities) as long as they fall within the time window. Such interaction will typically be an inhibition or only a small facilitation. This arrangement affords more flexibility in a complex environment. For example, response depression may occur with nearly simultaneous but distant stimuli, making it easier for the organism to focus attention on the more important event.

\section{Predictions}

The TWIN model makes a number of empirical predictions. First, the amount of multisensory integration should depend on the stimulus onset asynchrony (SOA) between the stimuli. Indeed, the effect tends to be most prominent when there is some characteristic temporal asynchrony between the stimuli (Colonius \& Arndt, 2001; Frens et al., 1995). Within the model this simply means that a stimulus with faster peripheral processing has to be delayed in such a way that the 


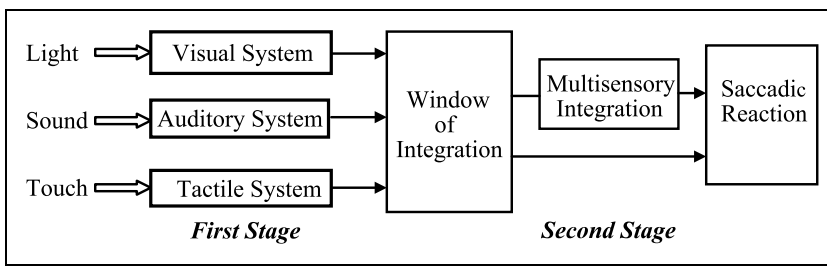

Figure 1. Two-stage schema for TWIN model. Multisensory integration occurs only if peripheral processes terminate within the temporal integration window (see text).

arrival times of both stimuli have a higher probability of falling into the window of integration.

Second, the probability of interaction, $\operatorname{Pr}[I]$, should depend on unimodal features that affect the speed of processing in the first stage, like stimulus intensity or eccentricity. For example, if a stimulus from one modality is very strong compared to the other stimulus' intensity, the former stimulus' peripheral processing speed will increase, and the chances that both peripheral processes terminate within the time window will be smaller (assuming simultaneous stimulus presentations). The resulting low value of $\operatorname{Pr}[I]$ is in line with the empirical observation that a very strong target signal will effectively rule out any further reduction of reaction time by adding a stimulus from another modality (e.g., Corneil, Wanrooij, Munoz, \& Van Opstal, 2002).

Finally, the amount of multisensory integration $(\Delta)$ and its direction (facilitation or inhibition) occurring in the second stage depends on crossmodal features of the stimulus set, in particular, spatial disparity and laterality (laterality here refers to whether or not all stimuli appear in the same hemisphere). Crossmodal features cannot have an influence on first stage processing time since the modalities are yet being processed in separate pathways. More specific predictions require an explication of the rules governing the window-of-integration mechanism in specific task requirements.

\section{Integration Rule Assumptions}

\section{Focused Attention Task}

When the task is to orient toward the target
stimulus ignoring stimuli from other modalities,
the first stage terminates when the target peripheral
process ends. Multisensory integration occurs only
if the nontarget stimulus wins the race in the first
stage opening a time window such that the
termination of the target peripheral process
is enclosed in the window.

In other words, in the focused attention situation, the window of integration is "opened" only by activity triggered by the nontarget stimulus, but first stage duration is determined by the target stimulus processing time regardless of the identity of the winner of the race.

\section{Redundant Target Task}

When the task is to orient toward the first stimulus detected no matter of which modality, the first stage duration is defined by the winner's peripheral processing time, and the window of integration is opened by whichever stimulus wins the race.

From these assumptions, further predictions concerning the effects of varying stimulus intensity follow. Take, for example, a focused attention task with a visual target and an auditory nontarget stimulus. Increasing the intensity of the visual stimulus will speed up visual peripheral processing (up to some minimum level), thereby increasing the chances for the visual target to win the race. Thus, the probability that the window of integration opens decreases predicting less multisensory integration. Increasing the intensity of the nontarget auditory stimulus, on the other hand, leads to the opposite prediction: The auditory stimulus will have a better chance to win the race and to open the window of integration, hence predicting more multisensory integration to occur on average.

Note that the principle of "inverse effectiveness," according to which multisensory facilitation is strongest when stimulus strengths are weak or close to threshold level (Meredith \& Stein, 1986a), can be accommodated in the model by the following additional hypothesis: For low-intensity stimuli, the window becomes larger so as to increase the likelihood of multisensory integration. ${ }^{1}$

\section{Separating Spatial and Temporal Factors of Integration}

Expected multisensory integration is defined as the difference between mean response time in the unimodal and the crossmodal conditions. An important property of the TWIN model, formally derived in the model section of the Appendix, is the factoring of expected multisensory integration, namely, expected multisensory integration is simply the product of the probability of interaction $\operatorname{Pr}[I]$ and the amount and sign of interaction $(\Delta)$. According to the assumptions, the first factor depends on the temporal configuration of the stimuli (SOA), whereas the second factor depends on their spatial configuration. Note that this separation of temporal and spatial factors is in accordance with the definition of the window of integration: The incidence of multisensory integration hinges upon the stimuli to occur in temporal closeness, whereas the amount and sign of interaction $(\Delta)$ is modulated by spatial proximity, reaching from enhancement for neighboring stimuli to possible inhibition for distant stimuli.

\section{EXPERIMENT}

The alignment of visual, auditory, and tactile topographical maps in the SC (cf. Meredith \& Stein, 1986a, 
1986b) suggests that, in analogy to visual-auditory interaction, visual-tactile interaction in saccade generation should depend on the spatial configuration of the stimuli. Here we examined the effect of a tactile stimulus (vibration applied to palm) on response time for saccades toward a visual target as a function of the spatial-temporal visual-tactile stimulus configuration. Visual and tactile stimuli were presented $20^{\circ}$ or $70^{\circ}$ left or right of the fixation point. Subjects were asked to make a saccade as quickly and as accurately as possible toward the visual stimulus appearing randomly left or right. They were instructed to ignore a tactile accessory stimulus presented at the corresponding hand position ipsi- or contralateral to the visual target either before the visual stimulus (100 or $50 \mathrm{msec}$ ), simultaneous to, or $50 \mathrm{msec}$ after the visual. In unimodal trials, only a visual stimulus was presented. They were randomly mixed with bimodal trials.

\section{RESULTS}

Mean RTs and standard errors over all subjects as a function of eccentricity and laterality are shown in Figure 2. If tactile stimulation had no effect on RT, all means within a given eccentricity $\left(20^{\circ}\right.$ or $\left.70^{\circ}\right)$ should be about the same. However, presentation of the tactile accessory stimulus had a facilitating effect on saccadic reaction time $(p<.01)$. Ipsilateral bimodal stimulation had a stronger effect than contralateral presentation $(p<.01)$, and responses to the $20^{\circ}$ position were faster than to the $70^{\circ}$ position $(p<.01)$. Furthermore, all SOAs had a significant effect on saccadic reaction time $(p<.01)$.

To better illustrate the effects of eccentricity and SOA on multisensory integration, the data are presented in terms of multisensory response enhancement (MRE) in analogy to Meredith and Stein (1986a) (cf. Colonius \& Diederich, 2002; Anastasio, Patton, \& BelkacemBoussaid, 2000):

$$
\mathrm{MRE}=\frac{R T_{\text {unimodal }}-R T_{\text {bimodal }}}{R T_{\text {unimodal }}} \times 100 .
$$

The following MRE values were obtained and presented in Table 1.

Multisensory integration, as measured by MRE, is a monotonically decreasing function of SOA. That is, in the given range of SOA values, multisensory integration is the larger the earlier the tactile stimulus is presented. Note that this is consistent with the TWIN model's prediction: The earlier the tactile nontarget is presented relative to the target, the higher its chance to win the peripheral race and to open the integration window. At $\mathrm{SOA}=50 \mathrm{msec}$, the tactile peripheral process terminates too late to open the window.

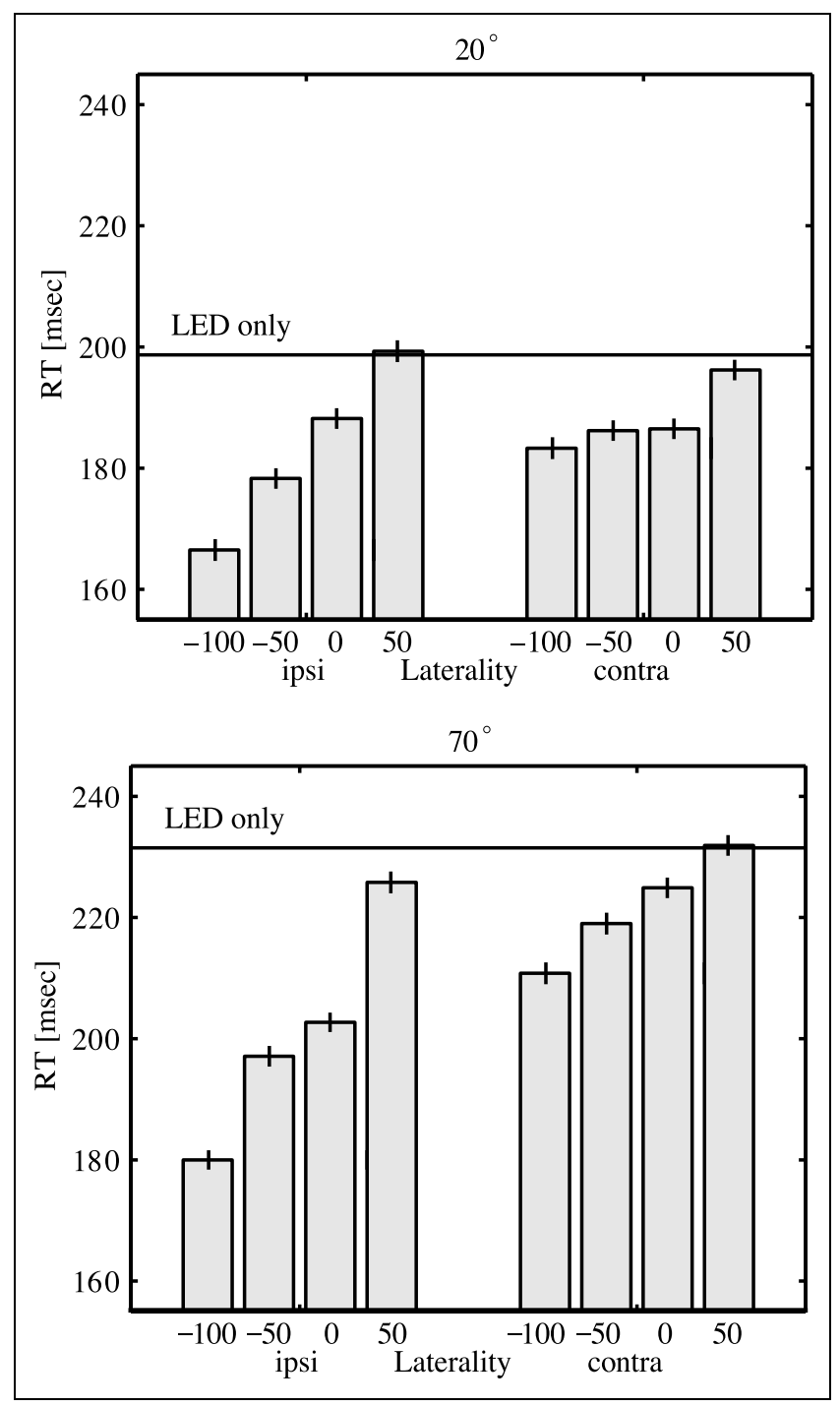

Figure 2. Mean saccadic RT (in msec) across all six participants for $20^{\circ}$ position (upper panel) and $70^{\circ}$ position (lower panel) of visual target, as a function of laterality and SOA. Unimodal visual RT (LED only) is indicated by the horizontal lines.

The fact that saccadic reaction time typically increases with eccentricity of the visual target (up to $30^{\circ}$, the increase is about $0.4 \mathrm{msec}$ per $1^{\circ}$; e.g., Findlay \& Walker, 1999) could be a confounding factor for the effect of eccentricity on facilitation. However, the relative enhancement as measured by the MRE introduced above would not be affected by this. Nonetheless, MRE increases with increasing eccentricity of the visual stimulus separately for the ipsilateral and the contralateral conditions (Table 1).

The error rates for moving the eyes to the nontarget direction are listed in parentheses in Table 1. As expected, this rate increases the earlier the nontarget is presented contralateral to the target, but for ipsilateral presentations erroneous responses are below $5 \%$ at $20^{\circ}$ and below $8 \%$ at $70^{\circ}$. 
Table 1. Multisensory Response Enhancement (MRE) (in $\mathrm{msec}$ ) as a Function of Eccentricity, SOA, and Laterality

\begin{tabular}{|c|c|c|c|c|}
\hline \multirow[b]{4}{*}{ SQA } & \multicolumn{4}{|c|}{ Eccentricity } \\
\hline & \multicolumn{2}{|c|}{$20^{\circ}$} & \multicolumn{2}{|r|}{$70^{\circ}$} \\
\hline & \multicolumn{2}{|c|}{ Laterality } & \multicolumn{2}{|c|}{ Laterality } \\
\hline & Ipsilateral & Contralateral & Ipsilateral & Contralateral \\
\hline-100 & $16.2(3.9)$ & $7.8(4.4)$ & $22.4(5.8)$ & $8.9(17.4)$ \\
\hline-50 & 10.3 (1.9) & $6.3(3.2)$ & $14.8(7.1)$ & $5.4(12.3)$ \\
\hline 0 & $5.3(3.9)$ & $6.2(2.7)$ & $12.4(6.1)$ & $2.9(9.3)$ \\
\hline 50 & $-0.3(2.9)$ & $1.3(2.4)$ & $2.5(5.2)$ & $-0.2(8.3)$ \\
\hline
\end{tabular}

For example, an MRE value of 10 means that saccadic reaction time to the visual target is reduced by $10 \%$ when a tactile stimulus is present.

\section{TWIN Model Fit}

According to the TWIN model, saccadic reaction time in the bimodal condition equals the sum of peripheral visual target processing time and the duration of the neural integration stage. Multisensory integration in the second stage occurs if (i) the race in the first stage is won by the tactile (nontarget) modality and (ii) termination of the visual peripheral processing time falls within the TWIN.

To allow for quantitative predictions, independent exponential peripheral processing times are assumed in the TWIN model. In total, there were nine parameters to be estimated from 18 data points (corresponding to the means observed in the different experimental conditions).

The fits, obtained separately for each subject, are illustrated in Figure 3 for two of the six subjects, the other fits being similar. ${ }^{2}$

Obviously, the model captures the main features of the data at the level of the means. Mean saccadic reaction time is increasing with SOA for all stimulus configurations, except for the $70^{\circ}$ contralateral condition in participant ML where monotonicity is reversed due to inhibition, consistent with the model. Moreover, the parameter estimates are ordered as expected for all participants (including those not reported here): (a) visual peripheral processing is faster for the $20^{\circ}$ position than for the $70^{\circ}$ position; (b) peripheral tactile processing is slower than visual processing; (c) the amount of facilitation is the larger the closer the tactile stimulus is to the visual. The optimal width for the time window was about 200 msec (Table 2).

\section{DISCUSSION}

\section{Experiment}

Saccadic reaction time to a visual target presented randomly left or right from fixation was reduced by up to

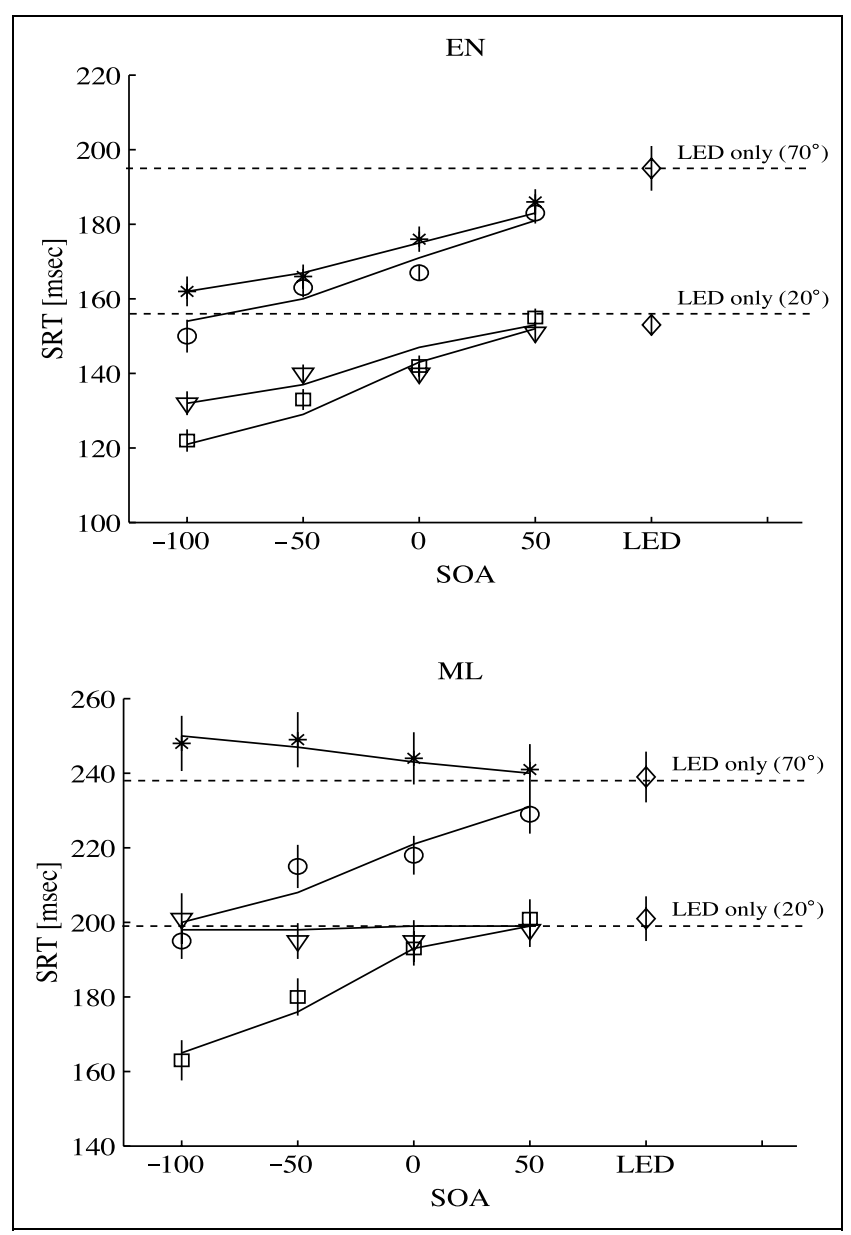

Figure 3. Fit of TWIN model mean saccadic RTs for participants EN and ML. Symbols refer to data, lines to predictions. Squares $=$ observed ipsilateral $20^{\circ}$; triangles $=$ observed contalateral $20^{\circ}$; circles $=$ observed $70^{\circ}$; and stars $=$ contralateral $70^{\circ}$. Horizontal lines refer to predicted unimodal saccadic RTs.

$22 \%$ when accompanied by a spatially nonpredictive tactile stimulus. This facilitation effect was larger for spatially aligned visual-tactile stimulus configurations than for contralateral presentation, and it increased with

Table 2. Parameter Estimates (in msec) for TWIN Model Processing Stages, Window Width, and Facilitation for Participants EN and ML

\begin{tabular}{lcc}
\hline Processing Time Estimates (msec) & $E N$ & $M L$ \\
\hline Visual peripheral $\left(20^{\circ}\right)$ & 38 & 13 \\
Visual peripheral $\left(70^{\circ}\right)$ & 75 & 52 \\
Tactile peripheral & 103 & 100 \\
Second stage & 118 & 186 \\
Integration window width & 201 & 200 \\
Facilitation ispilateral & 49 & 51 \\
Facilitation contralateral $20^{\circ} / 70^{\circ}$ & $33 / 39$ & $2 /-16$ \\
\hline
\end{tabular}


stimulus eccentricity $\left(20^{\circ}\right.$ vs. $\left.70^{\circ}\right)$. Moreover, responses were the faster the earlier the tactile stimulus was presented (in a range of the tactile preceding the visual by $100 \mathrm{msec}$ to following it by $50 \mathrm{msec}$ ). These results extend previous findings in our lab to nonsimultaneous visualtactile stimulus presentations (Diederich et al., 2003).

A partial explanation for the observed speed-up not involving any multisensory integration is that the tactile stimulus acts as a warning signal about the forthcoming visual target onset. In terms of the underlying neural oculomotor circuitry, a warning signal would lead to a decrease in activity of the fixation neurons in the SC, thereby facilitating saccade generation (cf. Frens et al., 1995). Note, however, that this would not account for the observation that ipsilateral stimulation was uniformly more effective than contralateral stimulation, because the tactile stimulus was not predictive with respect to the location of the target. Moreover, the differential effect of eccentricity on MRE cannot be explained with an invariant warning signal influence.

Although in line with recent results in Amlôt et al. (2003), the fact that even contralateral distractors had a facilitating effect on saccadic reaction time seems to be at odds both with certain empirical findings and with models of saccade target selection. A case in point is the "remote distractor" effect that consists in an increase of RT when the target and a distractor are presented at remote positions (Walker, Deubel, Schneider, \& Findlay, 1997), in accordance with a model by Trappenberg, Dorris, Munoz, and Klein (2001), holding that each stimulus produces an increase of collicular neuronal activity in separate populations in the collicular salience map. Target selection is accomplished by the existence of a single salience peak in the collicular map. This requires an inhibitory process taking a certain amount of time that is not needed in the single target case, contrary to the facilitation observed in our contralateral distractor conditions. It should be noted here, however, that the "remote distractor" effect seems to be confined to the visual modality. For example, both Colonius and Arndt (2001) and Frens et al. (1995) did find a facilitating effect of remote auditory distractors as well. While the size of these effects obviously depends on the relative intensity of target and distractor, it could be that visual distractors generate a more salient representation on the collicular activity map. This may be due in part to a more spreadout representation of auditory or tactile stimuli relative to visual stimuli, possibly resulting from differences in the corresponding RF sizes in the periphery.

Our results are compatible with the notion that the observed saccadic reaction time facilitation results from genuine visual-tactile integration taking place in the SC, an area involved in eye movement control. Stein and Meredith (1993, pp. 134-140) specify that an optimal interactive window for most visual-somatosensory neurons in cat SC appears to be less than $250 \mathrm{msec}$, which accords with our estimate of $200 \mathrm{msec}$. They estimate
65-100 msec for a visual stimulus to activate the neuron, while neural conduction time for a vibratory stimulus touching the skin takes some $40 \mathrm{msec}$ longer (Groh \& Sparks, 1996b). If similar numbers hold for the human brain, then maximal temporal overlap of discharges would be expected for SOAs from -100 to $-50 \mathrm{msec}$, the range were we actually did observe maximal response enhancement.

Note that the observed lack of contralateral inhibition on saccade generation discussed above is also at odds with the general finding of multisensory (visualauditory) "response depression" for spatially disparate stimuli measured both in SC neurons and overt orientation behavior in the cat (Wilkinson, Meredith, \& Stein, 1996; Stein, Meredith, Huneycutt, \& McDade, 1989). Interestingly, response enhancement under spatial disparity in an orienting task has also been found in peripheral sensory space of the cat (visual target at $\pm 30^{\circ}$ or $\pm 45^{\circ}$ ) when an auditory stimulus was more peripheral to the visual target (Jiang, Jiang, \& Stein, 2002). Moreover, multisensory integration properties of most SC neurons are mediated by influences from two cortical areas, the anterior ectosylvian sulcus and the rostral aspect of the lateral suprasylvian sulcus (Jiang, Wallace, Jiang, Vaughan, \& Stein, 2001; Wallace \& Stein, 1994). In the focused attention paradigm employed here, where target and nontarget modalities must be distinguished to accomplish the task, the contribution of further cortical areas making use of the warning signal function of the tactile stimulus cannot be ruled out as a partial explanation of the lack of contralateral inhibition.

Finally, note that the observed increase of MRE with the eccentricity of the visual target makes sense from a functional point of view. Orienting the eyes toward more eccentric targets takes more time (cf. Bell, Everling, \& Munoz, 2000), and thus a facilitating effect of the tactile accessory stimulus would be more important for more eccentric targets (see also Bell et al., 2001). This differential contribution of multisensory neurons is another instantiation of the principle of inverse effectiveness (see also Colonius \& Diederich, 2002; Anastasio et al., 2000).

\section{TWIN Model}

The TWIN model mechanisms postulated to generate the behavioral data are not meant to mirror the processes at the level of an individual neuron. There are many different types of multisensory convergence occurring in individual neurons (for a recent review, see Meredith, 2002) and some of their activities are consistent with the TWIN assumptions while others are not. For example, for certain neurons in cat SC, given a visual stimulus is always presented in the visual $\mathrm{RF}$ of the bimodal (visual-auditory) neuron, an auditory stimulus presented outside its RF will generate only re- 
sponse depression (or no interaction) regardless of the SOA between the stimuli (Stein \& Meredith, 1993, p. 140). This accords nicely with the separation of temporal and spatial factors postulated by the TWIN model. However, in other visual-auditory neurons, changing the temporal order and interval between the stimuli can change enhancement to depression even if their spatial arrangement is left invariant (Meredith, Nemitz, \& Stein, 1987). This latter type of behavior could only be accounted for in TWIN if two stimuli, after having passed the filter of the temporal window of integration, could still carry over information about their temporal distance to the subsequent convergence stage. Moreover, besides the common excitatoryexcitatory type of multisensory convergence, there is evidence for an excitatory-inhibitory type of neural circuit by which inputs from one modality inhibit those from the other (cf. Meredith, 2002). These circuits could play a specific role in focused attention situations (p. 37). In any event, at this stage of development the TWIN model operates at the level of behavioral data resulting from the combined activity of a possibly large number of neurons and from the specific task instructions, so that the existence of different types of multisensory convergence in individual neurons does not provide a strong model constraint.

Because stimulation from different modalities like vision and touch cannot interact (e.g., on the retina), the main assumption of the two-stage model, namely, the existence of a first stage of parallel independent modalityspecific activations in the afferent pathways, seems uncontroversial. It refers to a very early stage of processing where detection of the stimuli, but possibly no "higher" processes like localization and identification, takes place. Note that the two-stage assumption does not preclude the possibility of interaction between modality-specific pathways, nor between modality-specific and multisensory areas, at a later stage. In fact, there is increasing evidence that multisensory processing does not take place entirely in feedforward convergent pathways but that it can also modulate early cortical unisensory processing, as suggested by recent functional magnetic resonance imaging (fMRI) and ERP studies (see Laurienti et al., 2002; Macaluso, Frith, \& Driver, 2000, for fMRI; Molholm et al., 2002; McDonald, Teder-Sälejärvi, Di Russo, \& Hillyard, 2003, for ERP).

Even if certain assumptions of the TWIN model turn out to be inconsistent with an experimental result, one advantage of our model framework is that it facilitates the statement of clear-cut hypotheses about multisensory integration rules. For example, consider the "hypothesis of restricted influence." According to TWIN, unimodal stimulus properties, like intensity or spatial position, have a direct influence on first stage processing time. Moreover, unimodal properties may "indirectly" influence the resulting multisensory integration taking place in the second stage by modulating the opening of the integration window. The "restricted influence hypothesis" then denies existence of a direct influence of unimodal stimulus properties upon second stage processing. For example, increasing or decreasing stimulus intensity may not affect multisensory integration directly but only via changing properties of the window of integration (e.g., its probability of opening or its width). A saccadic eye movement study in the visual-auditory domain lends some support to this hypothesis. In a focused attention study, Arndt and Colonius (2003) found that saccadic response time decreased with increasing the intensity of a nontarget auditory stimulus while they could rule out any direct effect of intensity on second stage processing. ${ }^{3}$

Further experimental tests of the TWIN model should include a larger range of SOAs. There is some empirical evidence that the assumed symmetry of the crossmodal effects with respect to the temporal order in which the modalities are presented may not hold up in general (e.g., Corneil et al., 2002). Moreover, the second stage mechanisms should be specified in more detail with respect to the spatial stimulus configuration effects, possibly integrating aspects of the neural network models mentioned in the Introduction. There is a large database on RF properties of multisensory neurons available now (cf. Kadunce, Vaughan, Wallace, Benedek, \& Stein, 2001; Kadunce et al., 1997), and connecting these with behavioral data via an appropriate elaboration of the TWIN model should be a challenging task.

\section{METHODS}

\section{Participants}

Six students ( 4 women) served as paid voluntary participants in the experiments. All participants had normal vision. They were screened for their ability to follow the experimental instructions (proper fixation, few blinks during trial, saccades towards visual target). They gave their informed consent prior to their inclusion in the study.

\section{Apparatus and Stimulus Presentation}

Red light-emitting diodes (LED, $5 \mathrm{~mm}, 3.7 \mathrm{mcd}$ ) served as visual targets presented against a black background. An additional LED (red, $5 \mathrm{~mm}, 0.4 \mathrm{mcd}$ ) served as fixation point. Tactile stimuli were vibrations $(50 \mathrm{~Hz}$, $0.6 \mathrm{~V}, 1-2 \mathrm{~mm}$ amplitude) transmitted through wooden balls applied to the center of the palm, generated by two silenced oscillation devices (Mini-shaker, Type 4810 , B \& K). All stimuli were positioned on top of a table $(180 \times 130 \times 75 \mathrm{~cm})$ with a recess to sit in (referred to as the vertex). The fixation LED was $38.5 \mathrm{~cm}$ away from the lower edge of the table. For each experimental condition, the two vibrators were moved to the respective positions. 


\section{Experimental Procedure}

The experiment was carried out in a completely darkened room so that participants were unable to see their hands during the experiment. Every session began with 10 min of dark adaptation during which the measurement system was adjusted and calibrated. During this phase, the participants put their hands at the position used during the entire experimental block. Thus, the participants were aware of the hand position and, thus, the position of the tactile stimulus. Participants were sitting at the longitudinal side (at the vertex) using a chin rest facing the calibrating screen and wearing a video camera frame. Each trial began with the appearance of the fixation point. After a variable fixation time (800$1500 \mathrm{msec}$ ), the fixation point disappeared and both a visual and a tactile stimulus were presented for $500 \mathrm{msec}$ (no gap) at positions $20^{\circ}$ or $70^{\circ}$ left or right of the fixation point, the tactile stimulus $55 \mathrm{~cm}$ and the visual stimulus $50 \mathrm{~cm}$ away from the vertex. In unimodal trials, only a visual stimulus was presented. Subjects were instructed to move their eyes to the visual target as quickly as possible, while the tactile stimulus could be ignored. The interval between stimulus offset and fixation onset for the next trial was $2 \mathrm{sec}$. Each participant was first trained for 1000 trials not included in the data analysis.

\section{Data Collection}

Saccadic eye movements were recorded by an infrared video camera system (EyeLink system, Sensomotoric Instruments, Berlin, Germany) with temporal resolution of $250 \mathrm{~Hz}$ and horizontal and vertical spatial resolution of $0.01^{\circ}$. Eye position data from each trial were inspected for proper fixation at the beginning of the trial, for blinks, and for correct detection of start and endpoint of the saccade. Saccadic reaction time, start position of the eye, and end position after the saccade (vertical and horizontal positions in degree of visual angle relative to the straight ahead fixation point) were calculated from the controlled data samples.

\section{Experimental Design}

There were three levels of factor "laterality": ipsilateral, contralateral, and visual (LED) only. The second factor, "eccentricity," refers to the eccentricity of the visual and tactile stimuli with levels $20^{\circ}$ and $70^{\circ}$. The third factor, SOA, had levels $-100,-50,0$, and 50 msec. In each block of 270 trials, stimulus position $\left(20^{\circ}\right.$ or $\left.70^{\circ}\right)$ was held fixed resulting in 18 different conditions per block (four SOA levels, visual-tactile stimulus pairs presented ipsi- or contralateral, left or right, plus two unimodal visual conditions). Thus, except for the laterality, the participants knew where the stimuli would appear. There were seven blocks for either stimulus position resulting in 105 replications for each condition. RTs were then pooled over left/right presentation.

\section{Statistical Analysis}

A three-way ANOVA $(3 \times 2 \times 4)$ yielded significant main effects $(p<.01)$. Moreover, all interactions were significant $(p<.01$ for the pairs, $p<.05$ for the triple). Post hoc tests showed that the presence of a tactile accessory stimulus had a significant effect $(p<.01)$. Ipsi- versus contralateral bimodal stimulation was significant $(p<$ .01). Furthermore, all SOAs had a significant effect $(p<$ $.01)$ on RT as well as the eccentricity $(p<.01)$.

\section{APPENDIX}

\section{Quantifying Multisensory Integration in the TWIN Model}

According to the two-stage assumption, total RT in the crossmodal condition can be written as a sum of two random variables:

$$
R T_{\text {crossmodal }}=W_{1}+W_{2},
$$

where $W_{1}$ and $W_{2}$ refer to the first and second stage processing time, respectively. Let $I$ denote the event that multisensory integration occurs, having probability $\operatorname{Pr}(I)$. For the expected saccadic reaction time in the crossmodal condition then follows:

$$
\begin{aligned}
E\left[R T_{\text {crossmodal }]}=\right. & E\left[W_{1}\right]+E\left[W_{2}\right] \\
= & E\left[W_{1}\right]+\operatorname{Pr}[I] E\left[W_{2} \mid I\right] \\
& +(1-\operatorname{Pr}[I]) E\left[W_{2} \mid \text { not }-I\right] \\
= & E\left[W_{1}\right]+E\left[W_{2} \mid \text { not }-I\right]-\operatorname{Pr}[I] \\
& \times\left(E\left[W_{2} \mid \text { not }-I\right]-E\left[W_{2} \mid I\right]\right),
\end{aligned}
$$

where $E\left[W_{2} \mid I\right]$ and $E\left[W_{2} \mid\right.$ not $\left.-I\right]$ denote the expected second stage processing time conditioned on interaction occurring (I) or not occurring (not-I), respectively. Putting $\Delta \equiv E\left[W_{2} \mid\right.$ not $\left.-I\right]-E\left[W_{2} \mid I\right]$, this becomes

$$
E\left[R T_{\text {crossmodal }}\right]=E\left[W_{1}\right]+E\left[W_{2} \mid \text { not }-I\right]-\operatorname{Pr}[I] * \Delta .
$$

The term $\operatorname{Pr}[I] * \Delta$ can be interpreted as a measure of the expected saccadic $R T$ speed-up in the second stage with positive $\Delta$ values corresponding to facilitation, negative ones to inhibition. In the unimodal condition, no interaction is possible. Thus,

$$
E\left[R T_{\text {crossmodal }}\right]=E\left[W_{1}\right]+E\left[W_{2} \mid \text { not }-I\right],
$$

and

$$
E\left[R T_{\text {unimodal }}\right]=E\left[R T_{\text {crossmodal }}\right]=\operatorname{Pr}[I] * \Delta
$$

\section{TWIN Model for Visual-Tactile Interaction in Focused Attention}

With $V$ denoting visual peripheral processing time, $T$ tactile peripheral processing time, $\tau$ stimulus onset 
asynchrony, and $\omega$ the width of the integration window, multisensory integration depends on the event $I$ that the tactile stimulus wins the race in the first stage and that visual peripheral processing terminates within the window of integration,

$$
I=\{T+\tau<V<T+\tau+\omega\} .
$$

With $W_{2}$ denoting second stage processing time, expected saccadic response time to a visual target stimulus with tactile accessory presented at $\tau$ is

$$
E\left[R T_{V T, \tau}\right]=E[V]+E\left|W_{2}\right| n o t-I \mid-\operatorname{Pr}[I] * \Delta .
$$

As before, expected multisensory integration, measured as difference between expected uni- and bimodal response time, is

$$
\begin{aligned}
E\left[R T_{V}\right]-E\left[R T_{V T, \tau}\right] & =\operatorname{Pr}[I] *\left\{E\left[W_{2} \mid n o t-I\right]-E\left[W_{2} \mid I\right]\right\} \\
& =\operatorname{Pr}[I] * \Delta,
\end{aligned}
$$

where $E\left[R T_{V}\right]=E[V]+E\left[W_{2} \mid\right.$ not $\left.-I\right]$, the unimodal response time to the visual target only.

\section{Acknowledgments}

We thank B. E. Stein and two anonymous reviewers for helpful comments. This research was supported by grants from Deutsche Forschungsgemeinschaft SFB 517/C3 "Neurokognition" (HC) and Di 506/7-1, /7-2, /8-1 (AD).

Reprint requests should be sent to Hans Colonius, Department of Psychology, Oldenburg University, P.O. Box 2503, D-26111 Oldenburg, Germany, or via e-mail: hans.colonius@ uni-oldenburg.de.

\section{Notes}

1. In the focused attention task, the occurrence of inverse effectiveness should only depend on the intensity of the target stimulus. Although not implausible, this explanation of "inverse effectiveness" is post hoc and needs further empirical testing.

2. Given the large number of parameters and the relatively small dataset, we refrain from reporting quantitative fit statistics at this exploratory state of model testing.

3. A probability inequality test revealed that the amount of multisensory integration (as measured by comparison with the prediction of a simple, one-stage race model) was a function of the spatial visual-auditory configuration, but not of auditory intensity. Within the logic of the model, an auditory nontarget with higher auditory intensity level has a greater probability to open the time window, predicting a response speed-up. Because the auditory stimulus was not the target, this does not contradict the "inverse effectiveness" hypothesis.

\section{REFERENCES}

Amlôt, R., Walker, R., Driver, J., \& Spence, C. (2003).

Multimodal visual-somatosensory integration in saccade generation. Neuropsychologia, 41, 1-15.

Anastasio, T. J., Patton, P. E., Belkacem-Boussaid, K. (2000). Using Bayes' rule to model multisensory enhancement in the superior colliculus. Neural Computation, 12 , 1165-1187.

Arndt, A., \& Colonius, H. (2003). Two separate stages in crossmodal saccadic integration: Evidence from varying intensity of an auditory accessory stimulus. Experimental Brain Research, 148, 328-337.

Bell, A. H., Corneil, B. D., Meredith, M. A., \& Munoz, D. P. (2001). The influence of stimulus properties on multisensory processing in the awake primate superior colliculus. Canadian Journal of Experimental Psychology, 55, 123-132.

Bell, A. H., Everling, D., \& Munoz, D. P. (2000). Influence of stimulus eccentricity and direction on characteristics of pro- and antisaccades in non-human primates. Journal of Neurophysiology, 84, 2595-2604.

Colonius, H. (1990). Possibly dependent probability summation of reaction time. Journal of Mathematical Psychology, 34, 253-275.

Colonius, H., \& Arndt, P. (2001). A two-stage model for visual-auditory interaction in saccadic latencies. Perception and Psychophysics, 63, 126-147.

Colonius, H., \& Diederich, A. (2002). A maximum-likelihood approach to modeling multisensory enhancement. In T. G. Dietterich, S. Becker, \& Z. Ghahramani (Eds.), Advances in neural information processing systems 14, Cambridge: MIT Press.

Corneil, B. D., \& Munoz, D. P. (1996). The influence of auditory and visual distractors on human orienting gaze shifts. Journal of Neuroscience, 16, 8193-8207.

Corneil, B. D., Van Wanrooij, M., Munoz, D. P., \& Van Opstal, A. J. (2002). Auditory-visual interactions subserving goal-directed saccades in a complex scene. Journal of Neurophysiology, 88, 438-454.

Deneve, S., Latham, P, \& Pouget, A. (2001). Efficient computation and cue integration with noisy population codes. Nature Neuroscience, 4, 826-831.

Diederich, A. (1995). Intersensory facilitation of reaction time: Evaluation of counter and diffusion coactivation models. Journal of Mathematical Psychology, 39, 197-215.

Diederich, A., Colonius, H., Bockhorst, D., \& Tabeling, S. (2003). Visual-tactile spatial interaction in saccade generation. Experimental Brain Research, 148, 328-337.

Driver, J., \& Spence, C. (2000). Multisensory perception: Beyond modularity and convergence. Current Biology, 10, R731-R735.

Findlay, J. M., \& Walker, R. (1999). A model of saccade generation based on parallel processing and competitive inhibition. Behavioral and Brain Sciences, 22, 661-674.

Frens, M. A., \& Van Opstal (1998). Visual-auditory interactions modulate saccade-related activity in monkey superior colliculus. Brain Research Bulletin, 46, 211-224.

Frens, M. A., Van Opstal, A. J., Van der Willigen, R. F. (1995). Spatial and temporal factors determine auditory-visual interactions in human saccadic eye movements. Perception and Psychophysics, 57, 802-816.

Groh, J. M., \& Sparks, D. L. (1996a). Saccades to somotosensory targets. I. Behavioral characteristics. Journal of Neurophysiology. 75, 412-427.

Groh, J. M., \& Sparks, D. L. (1996b). Saccades to somotosensory targets: III. Eye-position-dependent somatosensory activity in primates superior colliculus. Iournal of Neurophysiology. 75. 439-453.

Harrington, L. K., \& Peck, C. K. (1998). Spatial disparity affects visual-auditory interactions in human sensorimotor processing. Experimental Brain Research, 122, 247-252.

Hughes, H. C., Nelson, M. D., \& Aronchick, D. M. (1998). Spatial characteristic of visual-auditory summation in human saccades. Vision Research, 38, 3955-3963.

Hughes, H. C., Reuter-Lorenz, P. A., Nozawa, G., \& Fendrich, R. (1994). Visual-auditory interactions in sensorimotor 
processing: Saccades versus manual responses. Journal of Experimental Psychology: Human Perception and Performance, 20, 131-153.

Jiang, W., Jiang, H., \& Stein, B. E. (2002). Two corticotectal areas facilitate orientation behavior. Journal of Cognitive Neuroscience, 14, 1240-1255.

Jiang, W., Wallace, M. T., Jiang, H., Vaughan, J. W., \& Stein, B. E. (2001). Two cortical areas mediate multisensory integration in superior colliculus neurons. Journal of Neuropbysiology. 85, 506-522.

Kadunce, D. C., Vaughan, J. W., Wallace, M. T., Benedek, G., \& Stein, B. E. (1997). Mechanisms of within-and cross-modality suppression in the superior colliculus. Lournal of Neurophysiology, 78, 2834-2847.

Kadunce, D. C., Vaughan, J. W., Wallace, M. T., \& Stein, B. E. (2001). The influence of visual and auditory receptive field organization on multisensory integration in the superior colliculus. Experimental Brain Research, 139, 303-310.

Laurienti, P. J., Burdette, J. H., Wallace, M. T. Yen, Y., Field, A. S., \& Stein, B.E. (2002). Deactivation of sensory-specific cortex by cross-modal stimuli. Journal of Cognitive Neuroscience, 14, 420-429.

Macaluso, E., Frith, C. D., \& Driver, J. (2000). Modulation of human visual cortex by crossmodal spatial attention. Science, 289, 1206-1208.

McDonald, J. J., Teder-Sälejärvi, W. A., Di Russo, F., \& Hillyard, S. A. (2003). Neural substrates of perceptual enhancement by cross-modal spatial attention. Journal of Cognitive Neuroscience, 15, 10-19.

Meredith, M. A. (2002). On the neuronal basis for multisensory convergence: A brief overview. Cognitive Brain Research, 14. 31-40.

Meredith, M. A., Nemitz, J. W., \& Stein, B. E. (1987). Determinants of multisensory integration in superior colliculus neurons: I. Temporal factors. Journal of Neuroscience. 7. 3215-3229.

Meredith, M. A., \& Stein, B. E. (1986a). Visual, auditory, and somatosensory convergence on cells in superior colliculus results in multisensory integration. Lournal of Neurophysiology, 56, 640-662.

Meredith, M. A., \& Stein, B. E. (1986b). Spatial factors determine the activity of multisensory neurons in cat superior colliculus. Brain Research, 365, 350-354.

Miller, J. (1982). Divided attention: evidence for coactivation with redundant signals. Cognitive Psychology, 14, 247-279.

Molholm, S., Ritter, W., Murray, M. M., Javitt, D. C., Schroeder, C. E., \& Foxe, J. J. (2002). Multisensory auditory-visual interactions during early sensory processing in humans: A high-density electrical mapping study. Cognitive Brain Research, 14, 115-128.
Pouget, A., Deneve, S., \& Duhamel, J. (2002). A computational perspective on the neural basis of multisensory spatial representations. Nature Reviews, 3, 741-747.

Raab, D. H. (1962). Statistical facilitation of simple reaction times. Transactions of the New York Academy of Sciences. 24. 574-590.

Robinson, D. A. (1972). Eye movements evoked by collicular stimulation in the alert monkey. Vision Research, 12, 1795-1808.

Sparks, D. L. (1986). Translation of sensory signals into commands for control of saccadic eye movements: Role of primate superior colliculus. Physiological Review, 66, 116-177.

Stein, B. E., Magalhaes-Castro, B., \& Kruger, L. (1976). Relationship between visual and tactile representations in cat superior colliculus. Journal of Neurophysiology, 39. 401-419.

Stein, B. E., \& Meredith, M. A. (1993). The merging of the senses. Cambridge: MIT Press.

Stein, B. E., Meredith, M. A., Huneycutt, W. S., \& McDade, L. (1989). Behavioral indices of multisensory integration: Orientation to visual cues is affected by auditory stimuli. Journal of Cognitive Neuroscience, 1, 12-24.

Trappenberg, T. P., Dorris, M. C., Munoz, D. P., \& Klein, R. M. (2001). A model of saccade initiation based on the competitive integration of exogenous and endogenous signals in the superior colliculus. Journal of Cognitive Neuroscience, 13, 256-271.

Wallace, M. T., Meredith, M. A., \& Stein, B. E. (1998). Multisensory integration in the superior colliculus of the alert cat. Journal of Neuropbysiology, 80, 1006-1010.

Wallace, M. T., \& Stein, B. E. (1994). Cross-modal synthesis in the midbrain depends on input from association cortices. Journal of Neurophysiology. 71, 429-432.

Wallace, M. T., Wilkinson, L. K., \& Stein, B. E. (1996). Representation and integration of multiple sensory inputs in primate superior colliculus. Journal of Neurophysiology. 76, 1246-1266.

Walker, R., Deubel, H., Schneider, W. X., \& Findlay, J. M. (1997). Effect of remote distractors on saccade programming: Evidence for an extended fixation zone. Journal of Neurophysiology, 78, 1108-1119.

Wilkinson, L. K., Meredith, M. A., \& Stein, B. E. (1996). The role of anterior ectosylvian cortex in cross-modality orientation and approach behavior. Experimental Brain Research, $112,1-10$.

Xing, J., \& Andersen, R. A. (2000). Models of the posterior parietal cortex which perform multimodal integration and represent space in several coordinate frames. Journal of Cognitive Neuroscience, 12, 601-614. 